\title{
Dermatitis Alérgica de Contacto por Corticoides
}

\author{
María Luisa Martínez Martíneza, Lorenzo Juan Pérez Garcíaa, Eduardo Escario Travesedoa, \\ María Rodríguez Vázqueza ${ }^{\text {, Guillermo Fernández Aragóna }}$
}

\begin{abstract}
a Servicio de Dermatología. Complejo Hospitalario Universitario de Albacete, España.

Correspondencia: María Luisa Martínez Martínez, Servicio de Dermatología, Complejo Hospitalario Universitario de Albacete, C/ Hermanos Falcó $n^{\circ}$ 37, 02006 Albacete, España. Correo electrónico: mlmartinezm@sescam.jccm.es.
\end{abstract}

Recibido el 20 de junio de 2009.

Aceptado para su publicación el 22 de agosto de 2009.

\begin{abstract}
RESUMEN
La dermatitis alérgica de contacto por corticoides representa un importante problema de diagnóstico clínico dado el cuadro de dermatosis crónica y atípica que puede originar. Para el diagnóstico disponemos de pruebas epicutáneas, las cuales presentan peculiaridades ya que el alergeno posee propiedades antiinflamatorias. Los corticoides se clasifican en diferentes grupos según su probabilidad de producir reacciones cruzadas. Este hecho nos permite elegir el tipo más adecuado en un determinado paciente alérgico. Presentamos una paciente con dermatitis alérgica de contacto a clobetasol 17 propionato, que refleja la dificultad de diagnóstico por su presentación como dermatosis paradójica.
\end{abstract}

Palabras clave. Dermatitis por Contacto. Corticosteroides. Pruebas del Parche.

\section{ABSTRACT}

Allergic contact dermatitis to corticosteroids

Allergic contact dermatitis to corticosteroids is a serious problem in clinical practice because of its chronic, atypical skin manifestations. Specific patch testing is used in diagnostic investigation, but the interpretation of the reactions may be difficult because of the anti-inflammatory properties of the corticosteroids. Corticosteroids are classified into different groups depending on the probability of allergic cross-reactivity between molecules. This classification enables us to choose the most suitable type for each specific patient. We present a patient with allergic contact dermatitis to topical clobetasol 17 propionate, which reflects the difficulty in diagnosing this paradoxical dermatosis.

Key words. Dermatitis, Contact, Corticosteroids, Patch tests.

\section{INTRODUCCIÓN}

La dermatitis alérgica de contacto es una respuesta de hipersensibilidad retardada a agentes exógenos. En las últimas décadas se ha producido un aumento en la incidencia de esta patología asociada a medicamentos de uso tópico, representando un importante problema de diagnóstico clínico en relación con su variabilidad en la expresión y los múltiples agentes etiológicos implicados. Entre los grupos alergénicos con mayor índice de sensibilización se encuentran los corticoides tópicos, siendo descrita la primera reacción de hipersensibilidad a corticoides en 1959 por Burckhardt. Desde entonces, son múltiples las publicaciones realizadas que avalan este hecho, estableciéndose una prevalencia de dermatitis alérgica de contacto por corticoides entre el $0,20 \%$ y el $5,98 \%$. Las causas que explican esta diferencia en cuanto a la prevalencia se relacionarían con los hábitos de prescripción, la concentración de corticoide, el vehículo utilizado, el conocimiento de la importancia de la sensibilización a los corticoides, la selección de los pacientes y la metodología de las pruebas epicutáneas.

\section{OBSERVACIONES CLÍNICAS}

Nuestra paciente es una mujer de 35 años, sin antecedentes patológicos ni alergias medicamentosas, que consultó por la aparición en región vulvar de placas eritematosas pruriginosas de aproximadamente un mes de evolución, negando aplicación de productos tópicos, exposición solar o toma de fármacos. No refirió antecedentes de patologías dermatológicas personales ni familiares.

A la exploración física se evidenciaron en región vulvar placas eritematosas, confluentes, de contornos mal definidos y no descamativas (figura 1). No aparición de vesícu- 
las ni ampollas. No signos de sobreinfección cutánea. No se objetivaron lesiones en mucosas oral ni genital, ungueales, capilares ni en el resto del tegumento.

Se realizó el diagnóstico de eccema subagudo de etiología irritativa, pautándose propionato de clobetasol. A la semana nuestra paciente acudió a la consulta por empeoramiento de su eccema vulvar junto a la aparición de lesiones pruriginosas en tronco. Éstas consistían en lesiones exudativas formadas por pápulas eritematosas, edematosas, distribuidas en tórax anterior y similares, aunque más confluen- tes, en espalda (figuras 2 y 3). Se realizaron pruebas epicutáneas con budesonida, tixocortol 21 pivalato e hidrocortisona 17 butirato, con lectura posterior a las 48 horas, 96 horas y 7 días. Se obtuvo un resultado positivo para hidrocortisona 17 butirato, que presenta reactividad cruzada con clobetasol 17 propionato.

Tras la retirada del corticoide se produjo la mejoría completa de las lesiones de la paciente, realizándose el diagnóstico de dermatitis alérgica de contacto por clobetasol 17 propionato bajo la expresión clínica de dermatosis paradójica.

\begin{tabular}{|c|c|c|c|}
\hline CLASE A & CLASE B & CLASE C & CLASE Da \\
\hline $\begin{array}{l}\text { Tipo: } \\
\text { Hidrocortisona }\end{array}$ & $\begin{array}{l}\text { Tipo: } \\
\text { Triancinolona acetónido }\end{array}$ & $\begin{array}{l}\text { Tipo: } \\
\text { Betametasona }\end{array}$ & $\begin{array}{l}\text { Tipo: } \\
\text { Hidrocortisona } 17 \text { butirato }\end{array}$ \\
\hline $\begin{array}{l}\text { Pivalato de tixocortol } \\
\text { Prednisolona } \\
\text { Metilprednisolona } \\
\text { Meprednisona } \\
\text { Prednisona }\end{array}$ & $\begin{array}{l}\text { Desonida } \\
\text { Amcinonida } \\
\text { Budesonida } \\
\text { Fluocinolona acetónido } \\
\text { Fluocinonida } \\
\text { Halcinonida }\end{array}$ & $\begin{array}{l}\text { Dexametasona } \\
\text { Fluocortolona } \\
\text { Fluocortina }\end{array}$ & $\begin{array}{l}\text { Betametasona } 17 \text { valerato (D1) } \\
\text { Prednicarbato (D2) } \\
\text { Aclometasona } 17 \text { propionato } \\
\text { Clobetasona } 17 \text { butirato (D1) } \\
\text { Hidrocortisona } 17 \text { valerato (D2) } \\
\text { Clobetasol } 17 \text { propionato (D1) } \\
\text { Betametasona dipropionato (D1) }\end{array}$ \\
\hline
\end{tabular}

Tabla 1. Grupos de reactividad cruzada entre corticoides tópicos. a El grupo D se divide en 2 subclases: D1 menos sensibilizante y D2 que sensibiliza con más frecuencia.

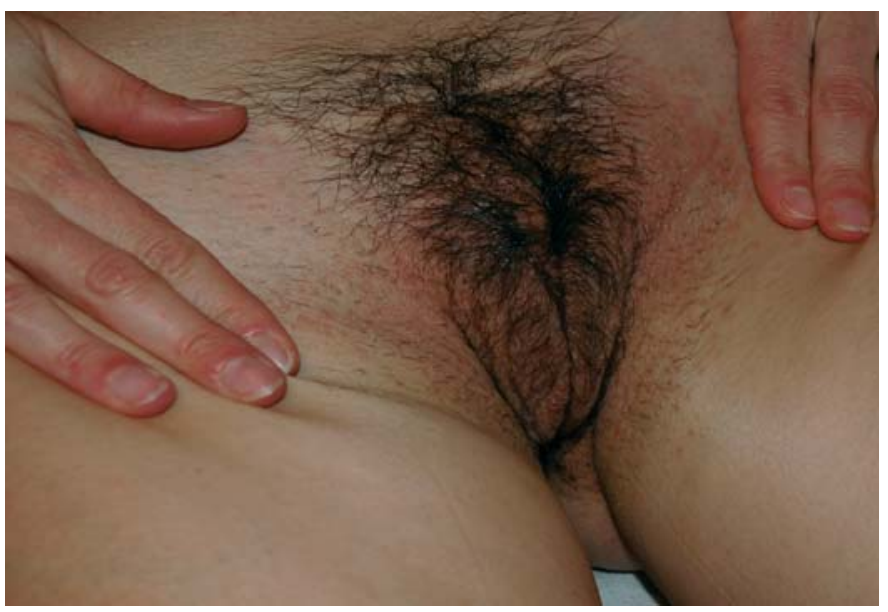

Figura 1. Eccema de contacto irritativo en región vulvar formado por placas eritematosas mal definidas y pruriginosas. 


\section{COMENTARIOS}

Los pacientes con dermatitis alérgica de contacto por corticoides suelen presentar lesiones eccematosas subagudas o crónicas de larga evolución, aunque en raras ocasiones pueden aparecer eccemas agudos exudativos. Algunos de estos pacientes refieren empeoramiento de su dermatitis con la administración de corticoides tópicos, aunque la mayoría presenta unas lesiones que, aunque no son exacerbadas por éstos, tampoco responden de manera adecuada a ellos. La causa de este eccema inespecífico, que oca- siona su infradiagnóstico, se encuentra en las propiedades antiinflamatorias y alergénicas simultáneas que poseen los corticoides, enmascarando la sensibilización. Existe una serie de factores de riesgo para esta patología que actuarían como indicadores de pacientes vulnerables, como son las úlceras en miembros inferiores, la dermatitis de éstasis, las dermatitis crónicas, la sensibilización a múltiples medicamentos, la dermatitis de las manos y la dermatitis atópica.

La dermatosis paradójica hace referencia a un cuadro clínico en el que aparecen lesiones eccematosas di-

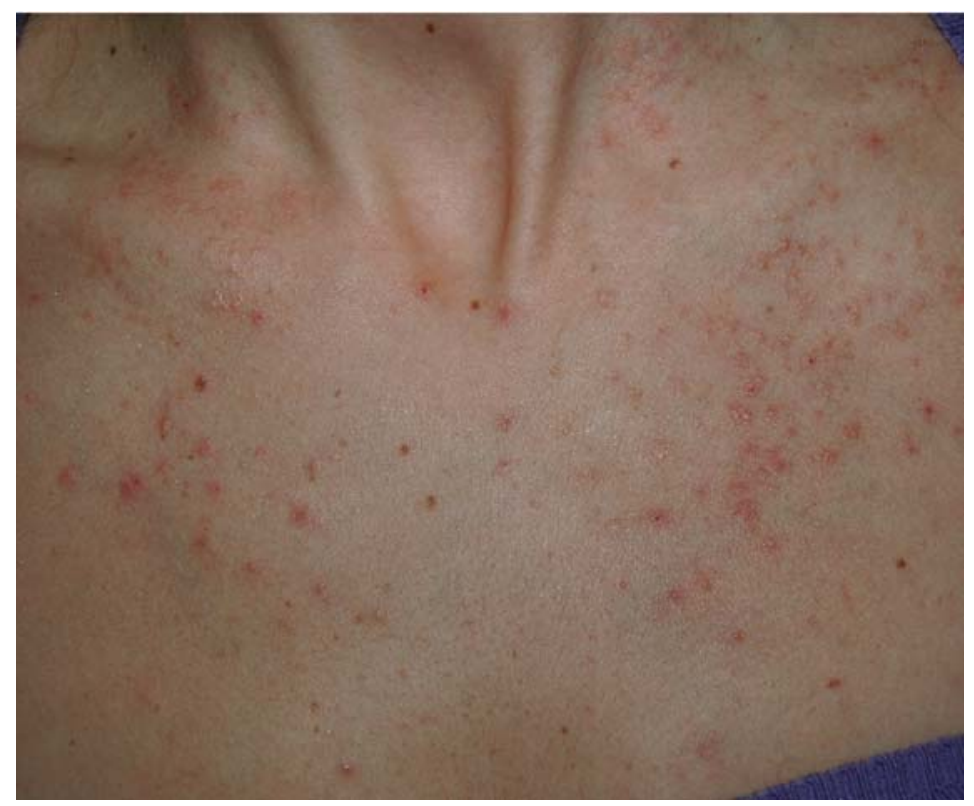

Figura 2. Lesiones exudativas papulosas eritematosas distribuidas en tórax anterior tras la aplicación de clobetasol.

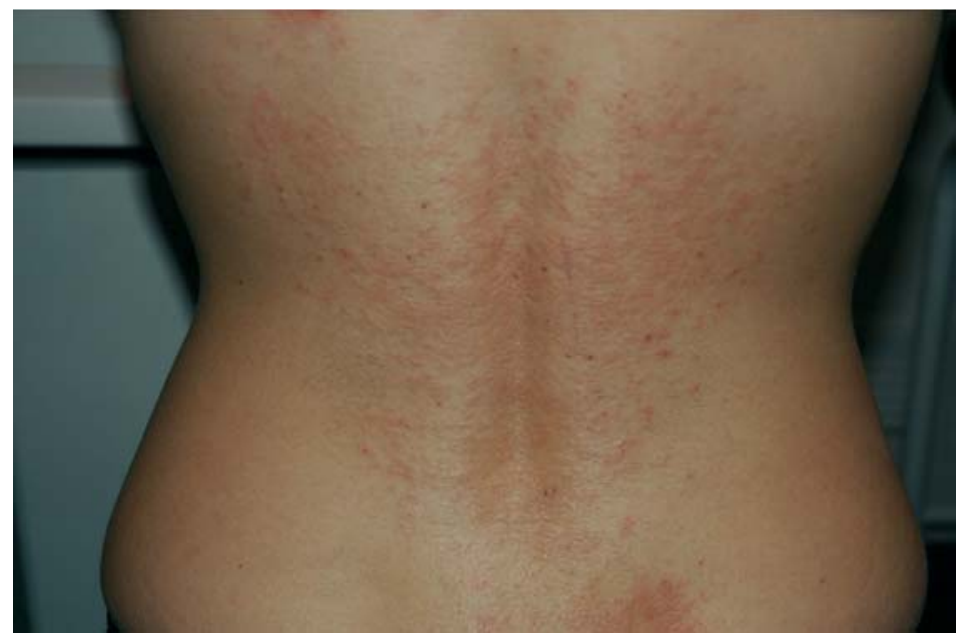

Figura 3. Lesiones papulosas eritematoedematosas confluentes en espalda tras aplicación de clobetasol. 
seminadas a distancia sin el desarrollo de lesiones en el lugar de aplicación. Este hecho está ampliamente descrito en la literatura con el uso de corticoides tópicos potentes capaces de suprimir la reacción local, gracias a su efecto antiinflamatorio, pero no la extensión a otras localizaciones.

La administración por vía sistémica de un corticoide responsable de una alergia de contacto puede producir una reactivación de la dermatitis en los lugares afectos, así como reacciones inmediatas que aunque raras pueden ser potencialmente graves como anafilaxia, urticaria, angioedema, púrpura y reacciones tipo eritema multiforme.

En la batería estándar de pruebas epicutáneas del Grupo Español de Investigación de Dermatitis de Contacto y Alergia Cutánea, los marcadores de alergia a esteroides son el pivalato de tixocortol, la budesonida y la hidrocortisona 17 butirato, que actuarían como prueba de cribado. Si obtenemos positividad con alguna de estas dos sustancias, debemos testar la batería de corticoides, o incluso si los resultados iniciales son negativos pero la sospecha es alta, ya que la negatividad de un marcador de grupo no excluye la sensibilización a alguno de los componentes de éste. Las reacciones positivas a las pruebas epicutáneas con corticoides aparecen a menudo como resultados inesperados en las pruebas de rutina, pero habitualmente son relevantes. La interpretación de los resultados se debe realizar con cautela, ya que estos fármacos pueden suprimir o retrasar la respuesta positiva. Por ello se realiza una tercera lectura a los 7 días, además de a las 46 y 96 horas, para evitar posibles falsos negativos. El "efecto borde" en las pruebas epicutáneas se observa sobretodo con corticoides de elevada potencia durante la primera lectura, consistiendo en la aparición de reacción únicamente en los bordes del parche, en relación con la menor concentración existente, en contraste con el centro, que tendría mayor concentración y por ello mayor efecto antiinflamatorio, y por tanto ausencia de respuesta.

Se han identificado cuatro clases de corticoides según su estructura química (tabla 1). Muchos de los pacientes sensibilizados a corticoides reaccionan a varios de ellos, debido a que los elementos que componen cada grupo tienen una estructura química similar, que explicaría la existencia de reacciones cruzadas entre ellos. La clasificación posee interés práctico, ya que determina qué corticoides puede utilizar el paciente y los que debe evitar según el grupo al que está sensibilizado. No obstante, se han descrito casos de reacciones cruzadas entre los grupos A-D, y más raramente entre B-D2.

En conclusión, los corticoides tópicos son fármacos ampliamente utilizados y de alto poder sensibilizante, por lo que es importante conocer la clínica que puede ocasionar una alergia a estos compuestos. Ante la falta de mejoría de una dermatosis crónica, y en presentaciones atípicas como la dermatosis paradójica que presentó nuestra paciente, se debe sospechar una dermatitis alérgica de contacto a corticoides para evitar posibles reacciones sistémicas y potencialmente graves que pueden desarrollar estos pacientes.

\section{BIBLIOGRAFÍA}

1. Jacob SE, Steele T. Corticosteroid classes: a quick reference guide including patch test substances and cross-reactivity. J Am Acad Dermatol 2006; 54:723-7.

2. Grupo Español de Investigación de Dermatitis de Contacto. Estudio epidemiológico de la dermatitis alérgica de contacto en España (2001). Actas Dermosifiliogr 2004; 95:14-24.

3. Davis MD, el-Azhary RA, Farmer SA. Results of patch testing to a corticosteroid series: a retrospective review of 1188 patients during 6 years at Mayo Clinic. J Am Acad Dermatol 2007; 56:921-7.

4. Pérez-Crespo M, Silvestre JF, Lucas A, Mataix J, Ballester I. Dermatitis de contacto por corticoides. Estudio retrospectivo de 3 años en una Unidad de Alergia Cutánea hospitalaria. Actas Dermosifiliogr 2009; 100:291-7.

5. Alcántara Villar M, Martínez Escribano J, López Sánchez JD, Frías Iniesta J, Pagán Alemán JA. Dermatitis de contacto por corticoides. Manejo clínico. Alergol Inmunol Clin 1999; 14:152-5.

6. Gómez-Vázquez M, Fernández-Redondo V, Toribio J. Dermatitis alérgica de contacto a tratamientos médicos tópicos. Actas Dermosifiliogr 2003; 94:150-4.

7. Laguna C, Zaragoza V, De la Cuadra J. Dermatitis de contacto alérgica a hidrocortisona como complicación del cuidado local de tatuajes. Actas Dermosifiliogr 2009; 100:235-48.

8. Pérez-Crespo M, Silvestre JF. Dermatitis de contacto alérgica por corticoides. Piel 2008; 23:415-20. 